PROCEEDINGS OF THE

AMERICAN MATHEMATICAL SOCIETY

Volume 130, Number 8, Pages 2377-2381

S 0002-9939(02)06359-1

Article electronically published on February 12, 2002

\title{
PERTURBATIONS OF SURJECTIVE CONVOLUTION OPERATORS
}

\author{
C. FERNÁNDEZ, A. GALBIS, AND D. JORNET
}

(Communicated by N. Tomczak-Jaegermann)

\begin{abstract}
Let $\mu_{1}$ and $\mu_{2}$ be (ultra)distributions with compact support which have disjoint singular supports. We assume that the convolution operator $f \rightarrow \mu_{1} * f$ is surjective when it acts on a space of functions or (ultra)distributions, and we investigate whether the perturbed convolution operator $f \rightarrow$ $\left(\mu_{1}+\mu_{2}\right) * f$ is surjective. In particular we solve in the negative a question asked by Abramczuk in 1984.
\end{abstract}

\section{INTRODUCTION}

For an element $\mu \in \mathcal{E}^{\prime}\left(\mathbb{R}^{N}\right)$, L. Ehrenpreis [5] and L. Hörmander 7] showed that the convolution operator $f \rightarrow \mu * f, f \in \mathcal{C}^{\infty}\left(\mathbb{R}^{N}\right)$ (resp. $f \in \mathcal{D}^{\prime}\left(\mathbb{R}^{N}\right)$ ) is surjective if and only if $\mu$ is slowly decreasing, that is, if its Fourier-Laplace transform $\hat{\mu}$ satisfies the following condition: There are constants $A, B, m$ such that

$$
\sup |\hat{\mu}(\xi)| \geq B\left(1+\left|\xi_{0}\right|\right)^{-m} \text { for all } \xi_{0} \in \mathbb{R}^{N},
$$

where the sup is taken over all $\xi \in \mathbb{R}^{N}$ satisfying $\left|\xi-\xi_{0}\right| \leq A \log \left(2+\left|\xi_{0}\right|\right)$. In $[\underline{8}$ Hörmander developed interesting and powerful methods with the aim of proving analogues of the theorem of supports of Titchmarch-Lions when the supports are replaced by the singular supports. As a corollary [8,5.4] he proved that $\mu_{1}+\mu_{2}$ is slowly decreasing whenever $\mu_{1}, \mu_{2} \in \mathcal{E}^{\prime}\left(\mathbb{R}^{N}\right)$ have disjoint singular supports and $\mu_{1}$ is slowly decreasing. In terms of the associated convolution operators this can be stated as follows: If $\mu_{1}, \mu_{2} \in \mathcal{E}^{\prime}\left(\mathbb{R}^{N}\right)$ have disjoint singular supports and $\mu_{1}$ defines a surjective convolution operator on $\mathcal{C}^{\infty}\left(\mathbb{R}^{N}\right)$ or on $\mathcal{D}^{\prime}\left(\mathbb{R}^{N}\right)$, then the convolution operator associated to $\mu_{1}+\mu_{2}$ is also surjective. In 1984 by also looking at the growth of the Fourier-Laplace transforms, Abramczuk [1] gave a direct proof of Hörmander's result, and he asked whether it remains true for convolution operators acting surjectively on the space of distributions of finite order $\mathcal{D}_{F}^{\prime}\left(\mathbb{R}^{N}\right)$.

In this paper we give a negative answer to the question posed by Abramczuk [1. Using recent results of Bonet, Galbis and Meise 2] we investigate perturbations of surjective convolution operators acting on spaces of ultradistributions of Beurling

Received by the editors July 24, 2000 and, in revised form, March 22, 2001.

2000 Mathematics Subject Classification. Primary 46F05; Secondary 46F10.

Key words and phrases. Convolution operator, slowly decreasing, ultradistributions.

This work was completed with the support of DGESIC under Proyecto PB97-0333.

The third author was also supported by Ministerio de Educación y Cultura, grant FP98 48285420

The authors want to express their gratitude to the referee for helpful suggestions. 
and of Roumieu type in the sense of Braun, Meise and Taylor [3]. In fact, we show that the result of Hörmander extends completely to convolution operators acting on $\mathcal{D}_{(\omega)}^{\prime}\left(\mathbb{R}^{N}\right)$ or on $\mathcal{D}_{\{\omega\}}^{\prime}\left(\mathbb{R}^{N}\right)$. We face the problem from a functional analytic point of view, and thus the role played by the disjointness of the supports is clarified. The behavior of the perturbed convolution operator on ultradifferentiable functions of Beurling or of Roumieu type is different. Whereas in the Beurling case the surjectivity is preserved, an example shows that this is not the case in the Roumieu setting.

\section{Preliminaries}

First we introduce the spaces of functions and ultradistributions and most of the notation that will be used in the sequel.

Definition 2.1. Let $\omega:[0, \infty[\rightarrow[0, \infty[$ be a continuous function which is increasing and satisfies $\omega(0)=0$ and $\omega(1)>0$. $\omega$ is called a weight function if it satisfies the following conditions:

( $\alpha) \omega(2 t) \leq K(1+\omega(t))$ for some $K \geq 1$ and for all $t \geq 0$.

(B) $\int_{1}^{\infty} \frac{\omega(t)}{t^{2}} d t<\infty$.

$(\gamma) \log (t)=o(\omega(t))$ as $t$ tends to $\infty$.

( $\delta) \varphi: t \rightarrow \omega\left(e^{t}\right)$ is convex.

Examples of weight functions can be found in 3 .

For a weight function $\omega$ we define $\tilde{\omega}: \mathbb{C}^{N} \rightarrow[0, \infty[$ by $\tilde{\omega}(z)=\omega(|z|)$ and again call this function $\omega$, by abuse of notation. The Young conjugate of $\varphi$ is defined by $\varphi^{*}(x)=\sup _{y>0}\{x y-\varphi(y)\}$.

Definition 2.2. Let $\omega$ be a weight function and let $\Omega$ be an open set in $\mathbb{R}^{N}$. We define

$$
\mathcal{E}_{(\omega)}(\Omega):=\left\{f \in C^{\infty}(\Omega):\|f\|_{K, \lambda}<\infty \text { for every } K \subset \subset \Omega \text { and every } \lambda>0\right\}
$$

and

$$
\begin{gathered}
\mathcal{E}_{\{\omega\}}(\Omega):=\left\{f \in C^{\infty}(\Omega): \text { for every } K \subset \subset \Omega \text { there exists } \lambda>0\right. \\
\text { such that } \left.\|f\|_{K, \lambda}<\infty\right\}
\end{gathered}
$$

where

$$
\|f\|_{K, \lambda}:=\sup _{x \in K} \sup _{\alpha \in \mathbb{N}_{0}^{N}}\left|f^{(\alpha)}(x)\right| \exp \left(-\lambda \varphi^{*}\left(\frac{|\alpha|}{\lambda}\right)\right) .
$$

$\mathcal{E}_{(\omega)}(\Omega)$ is endowed with its natural Fréchet topology, while $\mathcal{E}_{\{\omega\}}(\Omega)$ is a projective limit of $(L B)$ spaces.

The elements of $\mathcal{E}_{(\omega)}(\Omega)$ (resp. $\mathcal{E}_{\{\omega\}}(\Omega)$ ) are called $\omega$-ultradifferentiable functions of Beurling (resp. Roumieu) type. We write $\mathcal{E}_{*}(\Omega)$, where $*$ can be either $(\omega)$ or $\{\omega\}$. For a compact set $K$ in $\Omega$ we let

$$
\mathcal{D}_{*}(K):=\left\{f \in \mathcal{E}_{*}(\Omega): \operatorname{supp} f \subset K\right\},
$$

endowed with the induced topology. For $f \in \mathcal{D}_{*}(K)$ and $\lambda>0$ we will denote $\|f\|_{\lambda}=\|f\|_{K, \lambda}$ and

$$
\mathcal{D}_{*}(\Omega):=\operatorname{ind}_{j \rightarrow} \mathcal{D}_{*}\left(K_{j}\right)
$$

where $\left(K_{j}\right)_{j \in \mathbb{N}}$ denotes a fundamental sequence of compact sets of $\Omega$. 
The elements of $\mathcal{D}_{(\omega)}^{\prime}(\Omega)$ (resp. $\mathcal{D}_{\{\omega\}}^{\prime}(\Omega)$ ) are called $\omega$-ultradistributions of Beurling (resp. Roumieu) type.

Definition 2.3. Let $\mu \in \mathcal{E}_{*}^{\prime}\left(\mathbb{R}^{N}\right), \mu \neq 0$, be given. We define (compare with Braun, Meise and Taylor [3], Sect. 6)

$$
S_{\mu}: \mathcal{D}_{*}^{\prime}\left(\mathbb{R}^{N}\right) \longrightarrow \mathcal{D}_{*}^{\prime}\left(\mathbb{R}^{N}\right), S_{\mu}(E):=\mu * E,
$$

where $\langle\mu * E, \varphi\rangle=\langle E, \check{\mu} * \varphi\rangle$ and $\check{\mu} * \varphi: x \rightarrow \mu(\varphi(x+\cdot)), x \in \mathbb{R}^{N}$.

Then $S_{\mu}$ is a continuous linear operator.

We denote by $T_{\mu}: \mathcal{E}_{*}\left(\mathbb{R}^{N}\right) \longrightarrow \mathcal{E}_{*}\left(\mathbb{R}^{N}\right)$ the restriction of $S_{\mu}$ to $\mathcal{E}_{*}\left(\mathbb{R}^{N}\right)$.

Definition 2.4. Let $f \in \mathcal{D}_{*}^{\prime}\left(\mathbb{R}^{N}\right)$ be given. We write $\operatorname{sing}_{*} \operatorname{supp} f$ for the complement of the largest open set $\Omega$ such that $f \in \mathcal{E}_{*}(\Omega)$.

We will refer to $\operatorname{sing}_{*} \operatorname{supp} f$ as the $*$-singular support of $f$.

The following definition is a reformulation of the classical Ehrenpreis condition. We refer to [2, 2.3, 3.1].

Definition 2.5. (1) An element $\mu \in \mathcal{E}_{(\omega)}^{\prime}\left(\mathbb{R}^{N}\right)$ is called slowly decreasing for $(\omega)$ if there exists $C>0$ such that for each $x \in \mathbb{R}^{N}$ with $|x|>C$ there is $\xi \in \mathbb{C}^{N}$ with $|x-\xi| \leq C \omega(x)$ and $|\hat{\mu}(\xi)| \geq \exp (-C|\operatorname{Im} \xi|-C \omega(\xi))$.

(2) An ultradistribution $\mu \in \mathcal{E}_{\{\omega\}}^{\prime}\left(\mathbb{R}^{N}\right)$ is said to be slowly decreasing for $\{\omega\}$ if for each $m \in \mathbb{N}$ there exists $R>0$ such that for each $x \in \mathbb{R}^{N}$ with $|x|>R$ there exists $\xi \in \mathbb{C}^{N}$ satisfying $|x-\xi| \leq \frac{1}{m} \omega(x)$ such that $|\hat{\mu}(\xi)| \geq \exp \left(-\frac{1}{m} \omega(\xi)\right)$.

Remark 2.6. If $\mu \in \mathcal{E}_{*}^{\prime}\left(\mathbb{R}^{N}\right), \mu$ is slowly decreasing for $*$ if and only if the convolution operator $S_{\mu}$ is surjective [2, 2.9, 3.4].

\section{Results} 1].

We begin with a negative answer to the question posed by Abramczuk 1, remark

Theorem 3.1. There exists a distribution with compact support $\mu \in \mathcal{E}^{\prime}(\mathbb{R})$ whose support does not contain 0 and such that the convolution operator $S_{\delta+\mu}: \mathcal{D}_{F}^{\prime}(\mathbb{R}) \rightarrow$ $\mathcal{D}_{F}^{\prime}(\mathbb{R})$ is not surjective.

Proof. Let $\nu \in \mathcal{E}^{\prime}(\mathbb{R})$ be given such that the convolution operator $S_{\nu}$ is surjective on $\mathcal{D}^{\prime}(\mathbb{R})$ but does not admit a fundamental solution of finite order (see [6]). Let $R>0$ be such that $\operatorname{supp} \nu \subset[-R, R]$. We take $\varphi \in \mathcal{D}(\mathbb{R})$ identically 1 on a neighborhood of $[-(R+1), R+1]$. Let $E \in \mathcal{D}^{\prime}(\mathbb{R})$ be such that $S_{\nu}(E)=\delta$. We have

$$
S_{\nu}(\varphi E)=\delta-S_{\nu}((1-\varphi) E),
$$

from where it follows that $\mu:=-S_{\nu}((1-\varphi) E)$ has compact support. Moreover, 0 does not belong to the support of $\mu$. In fact, given $\psi \in \mathcal{D}(\mathbb{R})$, with support contained in $(-1,1)$, we have,

$$
\mu(\psi)=\left\langle-S_{\nu}((1-\varphi) E), \psi\right\rangle=-\langle(1-\varphi) E, \check{\nu} * \psi\rangle=0,
$$

since the support of $\check{\nu} * \psi$ is contained in $[-(R+1), R+1]$ and $1-\varphi$ is identically 0 on a neighborhood of this interval. Then

$$
S_{\delta+\mu}: \mathcal{D}_{F}^{\prime}(\mathbb{R}) \longrightarrow \mathcal{D}_{F}^{\prime}(\mathbb{R})
$$

cannot be surjective; indeed $S_{\delta+\mu}(f)=S_{\nu}((\varphi E) * f) \neq \delta$ for every $f \in \mathcal{D}_{F}^{\prime}(\mathbb{R})$, since $(\varphi E) * f \in \mathcal{D}_{F}^{\prime}(\mathbb{R})$ and $\nu$ does not admit a fundamental solution in $\mathcal{D}_{F}^{\prime}(\mathbb{R})$. 
In order to obtain positive perturbation results we show that the analogue of [7], 3.3] holds when we work with ultradistributions.

Lemma 3.2. Let $\omega$ be a weight function and let $\mu \in \mathcal{E}_{(\omega)}^{\prime}\left(\mathbb{R}^{N}\right)$ be given. Then the following are equivalent:

(1) $\mu$ is $(\omega)$-slowly decreasing.

(2) For each compact set $K$ in $\mathbb{R}^{N}$ there exist $m \in \mathbb{N}$ and $C>0$ such that $\sup _{x \in \mathbb{R}^{N}}|\varphi(x)| \leq C\|\mu * \varphi\|_{m}$ for all $\varphi \in \mathcal{D}_{(\omega)}(K)$.

(3) There exists a compact set $K$ in $\mathbb{R}^{N}$ with non-empty interior, $m \in \mathbb{N}$ and $C>0$ such that, for all $\varphi \in \mathcal{D}_{(\omega)}(K), \sup _{x \in \mathbb{R}^{N}}|\varphi(x)| \leq C\|\mu * \varphi\|_{m}$.

Proof. The equivalence between (1) and (2) is proved in [2, 2.6]. Clearly (2) implies (3).

To see that (3) implies (1), we first observe that without loss of generality we may assume that $K=\bar{B}_{r}$ for some $r>0$, where $B_{r}$ stands for the open ball around the origin of radius $r$. If $\mu$ is not $(\omega)$-slowly decreasing, we may proceed as in the proof of $(2) \Rightarrow(3)$ in [2 2.6] to find a sequence $\left(\nu_{j}\right)_{j \in \mathbb{N}}$ such that $\left(\mu * \nu_{j}\right)_{j \in \mathbb{N}}$ is bounded in $\mathcal{E}_{(\omega)}^{\prime}\left(\mathbb{R}^{N}\right)$, supp $\nu_{j} \subset \bar{B}_{r / 4}$ for every $j \in \mathbb{N}$, while $\left(\nu_{j}\right)_{j \in \mathbb{N}}$ is unbounded in $\mathcal{E}_{(\omega)}^{\prime}\left(\mathbb{R}^{N}\right)$. Therefore, we find $\varphi \in \mathcal{D}_{(\omega)}\left(B_{r / 2}\right)$ such that $\left(\nu_{j}(\varphi)\right)_{j \in \mathbb{N}}$ is an unbounded sequence in $\mathbb{R}$. Consequently $\left(\sup _{x \in \mathbb{R}^{N}}\left|\nu_{j} * \check{\varphi}(x)\right|\right)_{j \in \mathbb{N}}$ is not bounded, whereas $\left(\mu * \nu_{j} * \check{\varphi}\right)_{j \in \mathbb{N}}$ is bounded in $\mathcal{E}_{(\omega)}\left(\mathbb{R}^{N}\right)$, which contradicts $(3)$ since $\operatorname{supp} \nu_{j} * \check{\varphi}$ is contained in $\bar{B}_{r}$.

Theorem 3.3. Let $\omega$ be a weight function. Let $\mu_{1}, \mu_{2} \in \mathcal{E}_{(\omega)}^{\prime}\left(\mathbb{R}^{N}\right)$ be given, and assume that $S_{\mu_{1}}$ is surjective and that $\operatorname{sing}(\omega) \operatorname{supp} \mu_{1} \cap \operatorname{sing}(\omega) \operatorname{supp} \mu_{2}=\emptyset$. Then $S_{\mu_{1}+\mu_{2}}$ is surjective, too.

Proof. It is easy to deduce from the Paley-Wiener theorem [3, 3.4] that given $\eta \in$ $\mathcal{E}_{(\omega)}^{\prime}\left(\mathbb{R}^{N}\right)$ and a test function $\chi \in \mathcal{D}_{(\omega)}\left(\mathbb{R}^{N}\right)$, the sum $\eta+\chi$ is $(\omega)$-slowly decreasing if and only if $\eta$ is $(\omega)$-slowly decreasing. So we may assume that the supports are disjoint.

Let $K_{i}, i=1,2$, denote the support of $\mu_{i}, i=1,2$. Since $K_{1}$ and $K_{2}$ are disjoint compact sets in $\mathbb{R}^{N}$ we may take $\delta>0$ such that $\left(K_{1}+\bar{B}_{\delta}\right) \cap\left(K_{2}+\bar{B}_{\delta}\right)=\emptyset$. Then, for $\mu=\mu_{1}+\mu_{2}$ and $\varphi \in \mathcal{D}\left(\bar{B}_{\delta}\right)$,

$$
\|\mu * \varphi\|_{m}=\max \left(\left\|\mu_{1} * \varphi\right\|_{\mathrm{m}},\left\|\mu_{2} * \varphi\right\|_{\mathrm{m}}\right)
$$

which proves the result by Lemma 3.2

Remark 3.4. The classical case $\mathcal{C}^{\infty}\left(\mathbb{R}^{N}\right)$ is not a particular case of what we present here since $\omega(t)=\log \left(1+t^{2}\right)$ does not satisfy condition $(\gamma)$. However, the proof of Theorem 3.3 with Lemma 3.2 and [2, 2.9] replaced by [7, 3.2, 3.10] also works in this case.

As a corollary, we get the same result for ultradistributions of Roumieu type.

Corollary 3.5. Let $\omega$ be a weight function and let $\mu_{1}, \mu_{2} \in \mathcal{E}_{\{\omega\}}^{\prime}\left(\mathbb{R}^{N}\right)$ be given. If $S_{\mu_{1}}$ is surjective and $\operatorname{sing}\{\omega\} \operatorname{supp} \mu_{1} \cap \operatorname{sing}\{\omega\} \operatorname{supp} \mu_{2}=\emptyset$, then $S_{\mu_{1}+\mu_{2}}$ is surjective.

Proof. Again, it is enough to show that $\mu_{1}+\mu_{2}$ is $\{\omega\}$-slowly decreasing (see [2. 3.4]). According to [2, 3.2] there exists a weight function $\sigma$ with $\sigma=o(\omega)$ such that $\mu_{1}, \mu_{2} \in \mathcal{E}_{(\sigma)}^{\prime}\left(\mathbb{R}^{N}\right)$ and $\mu_{1}$ is $(\sigma)$-slowly decreasing. Observe that $\sigma=o(\omega)$ implies $\mathcal{E}_{\{\omega\}}\left(\mathbb{R}^{N}\right) \subset \mathcal{E}_{(\sigma)}\left(\mathbb{R}^{N}\right)[3,4.7]$, hence $\operatorname{sing}_{(\sigma)} \operatorname{supp} \mu_{i} \subset \operatorname{sing}_{\{\omega\}} \operatorname{supp} \mu_{i}, i=1,2$. 
Theorem 3.3 gives that $\mu_{1}+\mu_{2}$ is $(\sigma)$-slowly decreasing, and therefore it is $\{\omega\}$ slowly decreasing.

We investigate the surjectivity of the perturbed operator when it acts on functions. For the Roumieu case we make use of the characterization of surjective convolution operators on $\mathcal{E}_{\{\omega\}}(\mathbb{R})$ due to Braun, Meise and Vogt [4].

Theorem 3.6. (1) Let $\mu_{1}, \mu_{2} \in \mathcal{E}_{(\omega)}^{\prime}\left(\mathbb{R}^{N}\right)$ be given with disjoint $(\omega)$-singular supports. If $T_{\mu_{1}}$ is surjective on $\mathcal{E}_{(\omega)}\left(\mathbb{R}^{N}\right)$, so it is $T_{\mu_{1}+\mu_{2}}$.

(2) There exists $\mu \in \mathcal{E}_{\{\omega\}}^{\prime}(\mathbb{R})$ such that 0 does not belong to $\operatorname{supp} \mu$ and $T_{\delta+\mu}$ is not surjective on $\mathcal{E}_{\{\omega\}}(\mathbb{R})$.

Proof. (1) is an immediate consequence of Theorem 3.3 and 2, 2.9].

(2) By [4, 3.11] there is $\nu \in \mathcal{E}_{\{\sigma\}}^{\prime}(\mathbb{R})$ so that $S_{\nu}$ has an elementary solution $E$ but $T_{\nu}$ is not surjective. Then, choosing $\varphi \in \mathcal{D}_{\{\omega\}}(\mathbb{R}), \varphi=1$, in a neighborhood of $-\operatorname{supp} \nu$ and putting $\mu=-S_{\nu}((1-\varphi) E)$ as in the proof of Theorem 3.1 we obtain $T_{\delta+\mu}(f)=T_{\nu}((\varphi E) * f)$. Hence $T_{\delta+\mu}$ is not surjective, while supp $\delta \cap \operatorname{supp} \mu=$ $\emptyset$.

\section{REFERENCES}

1. W. Abramczuk, A class of surjective convolution operators, Pacific J. Math. 110 (1984) 1-7. MR 85k:46042

2. J. Bonet, A. Galbis, R. Meise, On the range of convolution operators on non-quasianalytic ultradifferentiable functions, Studia Math. 126 (1997) 171-198. MR 99a:46071

3. R. Braun, R. Meise, B.A. Taylor, Ultradifferentiable functions and Fourier analysis, Results Math. 17 (1990) 206-237. MR 91h:46072

4. R. Braun, R. Meise, D. Vogt, Existence of fundamental solutions and surjectivity of convolution operators on classes of ultradifferentiable functions, Proc. London Math. Soc. 61 (1990) 344-370. MR 91i:46038

5. L. Ehrenpreis, Solutions of some problems of division. Part IV, Amer. J. Math. 82 (1960) 522-588. MR 22:9848

6. L. Ehrenpreis, P. Malliavin, Invertible operators and interpolation in $\mathcal{A U}$ spaces, J. Math. Pures et Appl. 53 (1974) 165-182. MR 53:6316

7. L. Hörmander, On the range of convolution operators, Ann. Math. 76 (1962) 148-170. MR 25:5379

8. L. Hörmander, Supports and singular supports of convolutions, Acta Math. 110 (1963) 279302. MR 27:4070

Departamento de Análisis Matemático, Universidad de Valencia, Doctor Moliner 50, E-46100 Burjassot (VAlencia), Spain

E-mail address: Carmen.Fdez-Rosell@uv.es

Departamento de Análisis Matemático, Universidad de Valencia, Doctor Moliner 50, E-46100 Burjassot (Valencia), Spain

E-mail address: Antonio.Galbis@uv.es

Departamento de Matemática Aplicada, E.T.S. Arquitectura, Universidad Politécnica de Valencia, Camino de Vera, E-46071 Valencia, Spain

E-mail address: dajorca@mat.upv.es 